\title{
Pastos vivaces de los afloramientos ultrabásicos del NO de la Península Ibérica
}

\author{
Jesús Izco' \& Salvador Rivas-Martínez² \\ 'Departamento de Botánica, Universidad de Santiago de Compostela. E-15781, Santiago de Compostela. España. \\ ${ }^{2}$ Centro de investigaciones fitosociológicas, Madrid.
}

\section{Correspondencia}

J. Izco

e-mail: jesus.izco@usc.es

Recibido: 15 mayo 2017

Aceptado: 13 noviembre 2017

Publicado on-line: diciembre 2017

\begin{abstract}
Resumen
A partir de nuevos datos sobre los pastos de rocas ultrabásicas del NO de la provincia de La Coruña, se describe una nueva asociación y se reordena la sintaxonomía de este grupo de comunidades de la Península Ibérica, con la propuesta de la nueva alianza fitosociológica Saginion merinoi Izco \& RivasMartínez. Se propone Festuca actiophyta (M.I. Gut.) Izco \& Rivas Mart. stat. nov.
\end{abstract}

Palabras clave: Festucetea indigestae. Festuca actiophyta. Serpentinas y peridotitas. Riesgo de extinción.

\begin{abstract}
Northwestern Iberian ultramafic perennial grasslands

Based on new data about the grasses of ultrabasic rocks of the NW of the province of La Coruña, a new association is described, the syntaxonomy of this type of communities in the NW of the Iberian Peninsula is also reordered, and the new phytosociological alliance Saginion merinoi is proposed. Festuca actiophyta (M.I. Gut.) Izco \& Rivas Mart. stat. nov is proposed.
\end{abstract}

Key words: Festucetea indigestae. Festuca actiophyta. Risk of extinction. Serpentines and peridotites.

\section{Introducción}

La vegetación del noroeste ibérico sobre sustratos ultrabásicos fue inicialmente estudiada por Pinto da Silva (1965), en Portugal. En esta la publicación, propuso la asociación Armerietum eriophyllae Pinto da Silva 1965 para los pastos vivaces de las serpentinas del entorno de Vinhais y de Bragança. Más tarde, el mismo autor (Pinto da Silva, 1970) amplió su estudio a los afloramientos, con este tipo de rocas, en Morais, entre Maçedo de Cavaleiros y Mogadouro. Los inventarios de estas últimas localidades fueron impropiamente asimilados a la asociación Armerietum eriophyllae y el conjunto de los pastos vivaces ultrabásicos portugueses fueron agrupados en una asociación, bajo un nuevo nombre: Armerio (eriophyllae)Arenarietum fontqueri Pinto da Silva 1970, subordinada a la nueva alianza: Armerion eriophyllae Pinto da Silva 1970.

Nuevos estudios sobre estos pastos, en el macizo de Morais, municipio de Macedo de Cavaleiros (Aguiar et al., 1998), permitió individualizar las comunidades de este afloramiento ultrabásico bajo en nombre Arenario fontqueriArmerietum langei Aguiar, Penas \& Lousã 1998 e independizarlas de las descritas en el entorno de Bragança. Más tarde, la identificación en la comunidad de Armeria langei susbp. marizii (Daveau) Aguiar, Sánchez-Mata \& MonteiroHenriques dio lugar a la corrección del nombre de la asociación: Arenario fontqueri-Armerietum marizii Aguiar, Penas \& Lousã 1998 corr. Aguiar, Monteiro-Henriques \& Sánchez-Mata 2013 (Aguiar et al., 2013).

Por otro lado, Rodríguez-Oubiña \& Ortiz 
(1991) estudiaron la vegetación de las rocas ultrabásicas del noroeste ibérico: en las sierras de La Capelada y de Careón (Galicia, España), y Bragança y Mogadouro (Tras-os-Montes, Portugal). Como resultado, describieron para el conjunto de las rocas ultrabásicas gallegas una nueva asociación: Sagino merinoi-Plantaginetum radicatae Rodríguez-Oubiña \& Ortiz 1991 (tabla 1, con 24 inventarios). En ella distinguen la subasociación típica Rodríguez-Oubiña \& Ortiz 1991 (tab. 1, inventarios 1-19, holotypus invt, 2) y la subasociación asteretosum aragonensis Rodríguez-Oubiña \& Ortiz 1991 (tab. 1, inventarios 20-24, holotypus invt. 20).

\section{Objetivos}

Los estudios realizados se centran en los pastizales de las rocas ultrabásicas de la sierra de La Capelada (Cedeira, La Coruña), con objeto de describir la comunidad en la que se integra la festuca propia de esos medios, para la que se revisa su rango nomenclatural.

\section{Metodología}

\section{Espacio físico}

Los estudios realizados se centran en los pastizales con la festuca propia de Vixía Herbéira, sierra de La Capelada (Cedeira, La Coruña). Véase la nueva propuesta nomenclatural al final del texto.

La sierra de La Capelada se localiza junto al mar, con una altura de 613 m s.n.m., lo que le convierte en el segundo acantilado más alto de la Europa continental. En invierno, los vientos dominantes de sur y sudoeste traen frentes lluviosos con frecuencia, que descargan de forma generalizada en la sierra. En el verano, el desplazamiento del anticiclón de las Azores provoca vientos dominantes del noreste, también con lluvias frecuentes. Las temperaturas relativamente altas de la época estival provocan la evaporación del agua del mar y su ascenso sobre el acantilado hasta sobrepasar el muro litoral. En su ascenso, el vapor de agua se enfría a razón de valores medios entre 0’55 ㄷ/100 m (Aguiló et al., 1991) y de $0^{\prime} 59^{\circ} \mathrm{C} / 100 \mathrm{~m}$ (Carballeira et al., (1983), hasta rebasar las cumbres con temperaturas $3-4{ }^{\circ} \mathrm{C}$ inferiores a las de localidades costeras próximas. Además, al sobrepasar la línea de cumbres, el aire frío da lugar a lluvias, a las que se suman las nieblas frecuentes, que impiden la insolación y provocan un ambiente permanentemente húmedo y frío. Estas condiciones determinan un clima de tipo Hiperoceánico en la clasificación de RivasMartínez (2007).
Desde un punto de vista edáfico, La Capelada se caracteriza por su composición de rocas ultrabásicas, frecuentemente serpentinizadas (granulitas básicas y anfibolitas), con elevado contenido en $\mathrm{Mg}$, Fe, $\mathrm{Cr}$ y $\mathrm{Ni}$, pero pobres en $\mathrm{P}$ y K (Macías, 2014; Canosa, 2015). Los suelos originados a partir de estas rocas varían en función de la profundidad y de la vegetación que soportan. A los pequeños afloramientos rocosos se asocian leptosoles líticos. La Biogeografía y Bioclimatología siguen las propuestas de RivasMartínez (2004, 2007) y Rivas-Martínez et al. (2014 a, b). Los materiales se encuentran depositados en el herbario SANT.

\section{Resultados}

En la tabla 1 se reúnen cinco inventarios procedentes de puntos cercanos a la caseta de vigilancia de la costa de la sierra de La Capelada, todos ellos en el entorno de los 600 m.s.n.m., sobre suelos desnudos o incipientes. El número de especies por inventario oscila entre 12 y 17 , con una media de 14. Por lo general, la comunidad ocupa pequeñas áreas -apenas unos metros cuadradosen torno a los afloramientos rocosos y desaparece cuando los suelos se hacen más profundos, con pérdida de la influencia de la naturaleza de la roca madre y descenso del $\mathrm{pH}$ a valores ácidos. Estos procesos, unidos a la propia condición de la roca madre, se manifiestan en las tendencias tróficas de las especies de la comunidad: unas claramente ultrabásicas (Festuca actiophyta (M.I. Gut.) Izco \& Rivas Mart., Centaurea borjae Valdés Berm. \& Rivas Goday, Sagina merinoi Pau ex Merino, Asplenium adianthum-nigrum L. subsp. corunnense (Christ) Rivas Mart. -ésta, sobre la misma roca-), otro grupo son acidofilas - muestran preferencia por sustratos ácidos (Plantago radicata Hoffmmans \& Link, Glandora prostrata (Loisel.) DC. Thomas subsp. prostrata, Dantonia procumbens (Lam.) DC. in Lam. \& DC., Viola canina L., Erica mackaiana Bab. in J. Mackay), mientras que Erica vagans L. y Potentilla montana Brot. se comportan en el noroeste ibérico como ultrabásicas o basifilas.

\section{Discusión}

En la tabla adscrita a la asociación Sagino merinoi-Plantaginetum radicatae (RodríguezOubiña \& Ortiz, 1991) es evidente que existen dos grupos de inventarios claramente diferenciados. De un lado, los procedentes de los afloramientos ultrabásicos de la Dorsal Gallega (inventarios 1-11 y 20-24) y, de otro, de afloramientos de naturaleza 


\begin{tabular}{|c|c|c|c|c|c|}
\hline $\mathrm{N}^{\mathrm{a}}$ de inventario & 1 & 2 & 3 & 4 & 5 \\
\hline \multicolumn{6}{|l|}{ Área } \\
\hline \multicolumn{6}{|l|}{ Cobertura } \\
\hline $\mathrm{N}^{\circ}$ de especies & 13 & 15 & 17 & 13 & 12 \\
\hline \multicolumn{6}{|l|}{ Características de As. y rangos superiores } \\
\hline Festuca actiophyta & 4 & 3 & 4 & 3 & 3 \\
\hline Plantago radicata & 2 & + & . & . & 3 \\
\hline Sagina merinoi & . & 1 & 2 & + & . \\
\hline Centaurea borjae & . & . & 1 & . & 1 \\
\hline \multicolumn{6}{|l|}{ Compañeras } \\
\hline Scorzonera humilis & 2 & + & + & 2 & 1 \\
\hline Thymus praecox subsp. britannicus & 2 & 2 & 2 & 2 & 3 \\
\hline Centurea nigra subsp. rivularis & + & + & + & 1 & 1 \\
\hline Lotus corniculatus subsp. corniculatus & 1 & 2 & 2 & 1 & . \\
\hline Koeleria vallesiana & 1 & 2 & 1 & + & . \\
\hline Erica vagans & + & . & 1 & + & 1 \\
\hline Anthyllis vulneraria subsp. iberica & . & 1 & 1 & 1 & 1 \\
\hline Potentilla montana & 1 & 1 & 1 & . & . \\
\hline Leontadon taraxacoides & + & + & + & . & . \\
\hline Thymelaea coridifolia & + & + & . & . & 1 \\
\hline Carex flacca & + & . & 1 & . & 1 \\
\hline Glandora prostrata & . & 1 & 1 & + & . \\
\hline Linum catharticum & . & . & + & 1 & 1 \\
\hline Polygala vulgaris & . & + & . & + & . \\
\hline Danthonia decumbens & . & . & 1 & . & 1 \\
\hline Carex humilis & . & . & . & 2 & 2 \\
\hline Erica mackaiana & + & . & . & . & . \\
\hline Asplenium adianthum nigrum subsp. corunnense & . & + & . & . & . \\
\hline Viola canina & . & . & $r$ & . & . \\
\hline
\end{tabular}

Localidades. 1-5: Vixía Herbéira, Cedeira, La Coruña. Holotypus: tabla1, inventario 3.

análoga en la sierra de La Capelada (inventarios 12-19), estos análogos a los de nuestra tabla 1. A partir de estos últimos inventarios proponemos la asociación Festucetum actiophytae ass. nova, caracterizada por el Festuca actiophyta, Centaurea borjae, Euphorbia flavicoma DC. subsp. occidentalis M. Laínz.

Existen pues, al menos dos asociaciones de las rocas ultrabásicas gallegas: Sagino merinoiPlantaginetum radicatae Rodríguez-Oubiña \& Ortiz 1991 (p.p.) y Festucetum actiohytae Izco \& Rivas-Martínez ass. nova que muestran grandes diferencias con las comunidades portuguesas de Tras-os-Montes, sobre sustratos análogos, pero de ambiente mediterráneo, de las que difieren por su composición florística, series de vegetación en las que se integran, criterios que se expresan conjuntamente en la diferente adscripción corológica. Criterios que ya han sido avanzados parcialmente por Asensi et al. (2011) y Aguiar et al. (2013).

Ello permite configurar los pastos vivaces sobre sustratos ultramáficos del noroeste ibérico en dos alianzas diferentes. Las comunidades portuguesas se subordinan a la alianza Armerion eriophyllae Pinto da Silva 1970, mientras que las gallegas se integran en una nueva alianza: Saginion merinoi Izco \& Rivas-Martínez all. nova (Holotypus: Festucetum actiophytae).

Aparte las diferencias florísticas entre las dos 


\begin{tabular}{|c|c|}
\hline Alianza Armerion eriophyllae & Alianza Saginion merinoi \\
\hline $\begin{array}{l}\text { Alyssum serpyllifolium Desf. subsp. Iusitanicum T.R. Dudley \& P. } \\
\text { Silva }\end{array}$ & Agrostis truncatula subsp. commista Castrov. \& \\
\hline $\begin{array}{l}\text { Arenaria querioides Pourr. ex Willk. subsp. fontqueri (P. Silva) } \\
\text { Rocha Afonso }\end{array}$ & Alyssum serpyllifolium Desf. 'guitianae' \\
\hline Armeria eriophylla Willk. & Armeria merinoi (Bernis) Nieto Fel. \& Silva Pando \\
\hline Armeria langei Boiss. subsp. langei & Centaurea borjae Valdés Berm. \& Rivas Goday \\
\hline $\begin{array}{l}\text { Armeria langei subsp. marizii (Daveau) C. Aguiar, Sánchez-Mata } \\
\text { \& Monteiro-Heriques }\end{array}$ & Centaurea gallaecica (M. Laínz) Arnelas \& Devesa \\
\hline Avenula pratensis (L.) Dumort. subsp. Iusitanica F & Centaurea ultreiae Silva Pando \\
\hline $\begin{array}{l}\text { Dianthus laricifolius (Boiss. \& Reut.) subsp. marizii (Sampaio) } \\
\text { Franco }\end{array}$ & $\begin{array}{l}\text { Euphorbia flavicoma DC subsp. occidentalis M. } \\
\text { Laínz }\end{array}$ \\
\hline Festuca brigantina (Markgr.-Dann.) Markgr.-Dann. & Festuca actiophyta Izco \& Rivas Mart. \\
\hline Herr & Fritillaria nervosa Willd. subsp. falcata (Caballero) \\
\hline $\begin{array}{l}\text { Jasione sessiliflora Boiss. \& Reut. subsp. serpentinica (P. Silva) } \\
\text { Rivas Mart. \& Sánchez-Mata }\end{array}$ & Fernández-Arias \& Devesa \\
\hline Reseda virgata Boiss. \& Reuter & Sagina merinoi Pau ex Merino \\
\hline Silene legionensis L & Thlaspi alpestre Jacq. subsp. merinoi M. Laínz \\
\hline
\end{tabular}

Tabla 2. Características y diferenciales de las alianzas Armerion eriophyllae y Saginion merinoi. Table 2. Characteristics and differentials of the Armerion eriophyllae and Saginion merinoi alliances.

\begin{tabular}{|c|c|}
\hline Alianza Armerion eriophyllae & Ilianza Saginion merinoi \\
\hline $\begin{array}{l}\text { VPN: } \\
\text { Genisto hystricis-Quercetum rotundifoliae P. Silva } 1970 \\
\text { Pyro bourgeanae-Quercetum rotundifoliae Rivas- } \\
\text { Martínez } 1987 \\
\text { BOSQUES SECUNDARIOS: } \\
\text { Genisto hystricis-Quercetum rotundifoliae P. Silva } 1970 \\
\text { MATORRALES: } \\
\text { Lavandulo pedunculatae-Genistetum hystricis Rivas- } \\
\text { Martínez } 1968 \\
\text { Cisto ladaniferi-Genistetum hystricis P. Silva (1965) } \\
\text { 1970 } \\
\text { Genisto hystricis-Cytisetum multiflori Rivas-Martínez, } \\
\text { T.E. Díaz, F. Prieto, Loidi \& Penas } 1984\end{array}$ & $\begin{array}{l}\text { VPN: } \\
\text { Rusco aculeati-Quercetum roboris Br.-BI., P. Silva \& Rozeira } \\
1956 \\
\text { Blechno spicantis-Quercetum roboris Tüxen \& Oberdorfer } \\
1958 \\
\text { BOSQUES SECUNDARIOS: } \\
\text { Holco mollis-Betuletum celtibericae Amigo \& M.I. Romero } \\
2002 \\
\text { ORLAS FORESTALES Y MATORRALES: } \\
\text { Tamo communis-Rubetum ulmifoli Tüxen \& Oberdorfer } 1958 \\
\text { Cytisetum striati Bellot \& Casaseca ex Castroviejo } 1973 \\
\text { Ulici europaei-Cytisetum ingramii Rivas-Martínez } 1978 \\
\text { Ulici latebracteati-Cytisetum striati Rivas-Martínez ex J.C. } \\
\text { Costa, Izco, Lousā, Aguiar \& Capelo in J.C. Costa, Capelo, } \\
\text { Lousā, Antunes, Aguiar, Izco \& Ladero 2000 } \\
\text { Ulici izcoi-Ericetum cinereae Bellot } 1949 \text { corr. Rivas-Martínez, } \\
\text { Izco, Amigo \& Pulgar in Rivas-Martínez \& col. } 2011 . \\
\text { Cirsio filipenduli-Ericetum ciliaris Br.-BI., Pinto \& Rozeira } \\
\text { 1965 }\end{array}$ \\
\hline
\end{tabular}

Tabla 3. Series de vegetación a las que se vinculan las comunidades de las alianzas Armerion eriophyllae y Saginion merinoi. VPN: Vegetación potencial natural. Table 3. Vegetation series to which the communities of the Armerion eriophyllae and Saginion merinoi belong. VPN (= PNV): Potential Natural Vegetation. 
alianzas que se manifiestan en la tabla 2, tiene también valor discriminante la presencia en la alianza Armerion eriophyllae los numerosos terófitos, ausentes de la alianza Saginion merinoi: Aira cupaniana Guss., Allium guttatum Steven. subsp. sardoum (Moris) Stearn, Crucianella angustifolia L., Filago minima (Sm.) Pers., Galium parisiense L., Linaria aeruginea (Gouan) Cav. subsp. aeruginea, Linaria amethystea (Vent.) Hoffmanns \& Link subsp. amethystea, Micropyrum tenellum (L.) Link, Molineria laevis (Brot.) Asch. \& Graebn., Petrorhagia nanteuilii (Burnat) P.W. Ball \& Heywood, Sedum amplexicaule DC., Trifollium scabrum L., Trisetaria ovata (Pers.) Paunero, Tuberaria guttata (L.) Fourn., todos ellos de óptimo mediterráneo.

Las comunidades de las alianzas Armerion eriophyllae y Saginion merinoi se integran en conjuntos de vegetación completamente diferentes, que corresponden a dos series de vegetación, en el caso de la primera alianza, ligados a bosques perennifolios de Quercus rotundifolia Lam. y $Q$. suber $L$., la segunda a bosques caducifolios de Quercus robur L. (tab. 3).

La biogeografía o tipología jerárquica de los territorios en los que se localizan las diferentes comunidades, también señalan la independencia de las dos alianzas (tab. 4).

Entre la flora que define la asociación Festucetum actiophytae se cuentan algunos táxones incluidos en la lista roja de la flora vascular española: Festuca brigantina subsp. actiophyta, en la categoría Vulnerable (VU), en función de los criterios B1ab(iii)+2ab(iii) (Gómez-Orellana \& Ortíz,
2004; Moreno, 2008) y, como Festuca brigantina, en la misma categoría, en función del criterio B2ab(iii), en la lista de especies amenazadas de la UICN (Gómez-Orellana et al., 2011); por lo que respecta a Centaurea borjae, está catalogada como En peligro (EN), por los criterios $\mathrm{B} 1 \mathrm{ab}$ (iii)+2ab(iii), D2 (Gómez-Orellana, 2004; Moreno, 2008); la especie tiene la misma categoría en la lista de la UICN, basada en los criterios B1ab (iii)+2ab(iii) (GómezOrellana, 2011). La propia Festucetum actiophytae, tiene un área de presencia entre los $100-500 \mathrm{~km}^{2}$, por tanto, con un riesgo de extinción alto (HR) en la clasificación de Izco (2015), no consolidada, a falta de su análisis detallado. Esta comunidad de pastos forma parte de los hábitats 1230: Acantilados con vegetación de las costas atlánticas y bálticas (Véase López-Bedoya \& Pérez-Alberti, 2009) y 8220: Pendientes rocosas silíceas con vegetación casmofítica (Véase Pérez-Alberti \& López-Bedoya, 2009).

\section{Conclusiones}

Se propone una nueva asociación: Festucetum actiophytae, de las rocas ultrabásicas de la sierra de La Capelada (La Coruña), y una nueva alianza, Saginion merinoi, que agrupa las comunidades sobre ese tipo de rocas de los sectores corológicos Galaico-Portugués (distritos Compostelano interior y Galaico septentrional).

La asociación Festucetum actiophytae alberga algunos taxones incluidos en la Lista Roja 2010 de la flora vascular española, y ella misma es una comunidad en riesgo alto de extinción.

\begin{tabular}{|c|c|}
\hline Alianza Armerion eriophyllae & Allianza Saginion merinoi \\
\hline Región Mediterránea & Región Eurosiberiana \\
Subregión Mediterránea Occidental & Subregión Atlántico-Centroeuropea \\
Provincia Mediterránea Ibérica Occidental & Provincia Atlántico-Europea \\
Subprovincia Carpetano-Leonesa & Subprovincia Cantabroatlántica \\
Sector Lusitano-Duriense & Sector Galaico-Portugués \\
Distritos Braganzano y Ribaduriense & Distritos Brigantino y Compostelano interior \\
& Sector Galaico-Asturiano \\
& Distrito Galaico septentrional \\
\hline
\end{tabular}

Tabla 4. Unidades biogeográficas en las que se vinculan las comunidades de las alianzas Armerion eriophyllaey Saginion merinoi, según categorización biogeográfica de Rivas-Martínez et al. (2014 a, b). Table 4. Biogeographic units linking the communities of the Armerion eriophyllae and Saginion merinoi alliances, according to the biogeographical categorization of Rivas-Martínez et al. (2014 a, b). 


\section{Propuesta sintaxonómica}

Clase Festucetea indigestae Rivas Goday \& RivasMartínez 1971

Orden Jasiono sessiliflorae-Koelerietalia crassipedis Rivas-Martínez \& Cantó 1987

All. Armerion eriophyllae Pinto da Silva 1970

- Ass. Arenario fontqueri-Armerietum marizii Aguiar, Penas \& Lousã 1998 corr. Aguiar, MonteiroHenriques \& Sánchez-Mata 2013 (= Arenario fontqueri-Armerietum langei Aguiar, Penas \& Lousã 1998)

Pastizales mediterráneos sobre sustratos ultrabásicos. Distrito Ribaduriense

- Ass. Armerietum eriophyllae Pinto da Silva 1965 (= Armerio eriophyllae-Arenarietum fontqueri Pinto da Silva p.p. (excluidos los inventarios del macizo de Morais)

Pastizales mediterráneos sobre serpentinas. Distrito Braganzano

All. Saginion merinoi Izco \& Rivas-Martínez 2017 All. nova

(Holotypus: Festucetum actiophytae Izco \& RivasMartínez hoc loco)

- Ass. Festucetum actiophytae Izco \& RivasMartínez 2017 ass. nova. Holotypus: tabla 1, inventario 3 (= Sagino merinoi-Plantaginetum radicatae Rodríguez-Oubiña \& Ortiz 1991 p.p. (Tabla 1, inventarios12-19)

Pastizales eurosiberianos sobre sustratos ultrabásicos. Distrito Galaico Septentrional

- Ass. Sagino merinoi-Plantaginetum radicatae Rodríguez-Oubiña \& Ortiz 1991 p.p. (Tabla 1, inventarios 1-11 y 20-24)

Pastizales eurosiberianos sobre sustratos ultrabásicos. Distritos Brigantino y Compostelano Interior

\section{New floristic proposal}

De acuerdo con los caracteres diagnósticos aportados por Gutiérrez-Villarías et al. (1997): tallos ásperos, piezas del antecio muy vellosas, lema ovado-acuminada y colénquima de las láminas foliares discontinuo, junto a su comportamiento fitosociológico, con valor taxonómico (Izco, 1980), se propone un nuevo estatus taxonómico para la festuca de los afloramientos ultrabásicos de La Capelada, en el noroeste de la provincia de La Coruña: Festuca actiophyta (M.I. Gut.) Izco \& Rivas Mart. stat nov. [Basionym: Festuca brigantina (Markgr.-Dann.) Markgr.-Dann. subsp. actiophyta M.I. Gut. in Bot. J. Lin. Soc., 123 (3): 252-254. 1997].

\section{Referencias}

Aguiar, C., Monteiro-Henriques, T. \& Sánchez-Mata, D. (2013). New contributions on the flora and vegetation of Northeastern Portugal ultramafic outcrops. Lazaroa, 34, 141-150. http://dx.doi/10.5209/rev_LAZA.2013. v34.n1.43642.

Aguiar, C., Penas, A., Costa, J.C. \& Lousã, M. (1998). Vegetación endémica, no rupícola, de las rocas ultrabásicas de Trás-os-Montes (NE de Portugal). Itinera Geobot., 11, 249-262.

Aguiló, A.M., Aramburu, M.M.P., Ayuso, C.E., Blanco, A.A., Calatayud, P.T. et al. (1991). Guía para la elaboración de estudios del medio físico: Contenido y Metodología. Secretaría General Técnica, Ministerio de Obras Públicas y Transportes. Madrid. $572 \mathrm{pp}$.

Asensi, A., Aguiar, C., Sánchez-Mata, D. \& MonteiroHeriques, T., eds., (2011). Flora and vegetation of Iberian ultramafics. Excursion Guide. 7th. International Conference on Serpentine Ecology. Inst. Politécnico de Bragança. Portugal.

Canosa, F. (2015). Guía geológica de siete georutas en el concello de Cedeira. Concello de Cedeira, Publicacións municipáis. Cedeira. 70 pp.

Carballeira, A., Devesa, C., Retuerto, R., Santillán, E. \& Uceda, F. (1983). Bioclimatología de Galicia. Edit. Fundación Pedro Barrié de la Maza. Conde Fenosa. A Coruña. $591 \mathrm{pp}$.

Gómez-Orellana, L. (2004). Centaurea borjae. En A. Bañares, G. Blanca, J. Güemes, J.C. Moreno \& S. Ortiz (Eds.), (2004). Atlas y Libro Rojo de la Flora Vascular Amenazada de España. Dirección General de Conservación de la Naturaleza. Madrid, 1.069 pp.

Gómez-Orellana, L. (2011). Centaurea borjae. The IUCN Red List of Threatened Species 2011. e.T162359A5579170. http://dx.doi.org/10.2305/IUCN. UK.2011-1.RLTS.T162359A5579170.en. Descargado el 07 de febrero, 2017.

Gómez-Orellana, L. \& Ortiz, S. (2004). Festuca brigantina subsp. actiophyta. En A. Bañares, G. Blanca, J. Güemes, J.C. Moreno \& S. Ortiz (Eds.), (2004). Atlas y Libro Rojo de la Flora Vascular Amenazada de España. Dirección General de Conservación de la Naturaleza. Madrid, 1.069 pp.

Gómez-Orellana, L., Ortiz, O. \& Caldas, F.B. (2011). Festuca brigantina. The IUCN Red List of Threatened Species 2011. e.T162389A5584457. http://dx.doi.org/10.2305/IUCN.UK.2011-2.RLTS. T162389A5584457.en. Descargado el 07 de febrero, 2017.

Gutiérrez Villarías, I., Romero, M.I., Soñora, X. \& Homet, J. (1997). A new subspecies of Festuca Brigantina (Markgr.-Dann.) Markgr.-Dann. Bot. J. Lin. Soc. 123 (3), 249-255. doi: 10.1111/j.1095-8339.1997. tb01417.x

Izco, J. (1980). The role of phytosociological data in floras and taxonomy. Bot. J. Lin. Soc. 80, 179-190. doi:10.1111/j.1095-8339.1980.tb01664.x

Izco, J. (2015). Risk of extinction of plant communities: Risk and assessment categories. Pl. Biosyst., 149 (3), 589-602. doi/10.1080/11263504.2014.1000998 
López-Bedoya, J. L. \& Pérez-Alberti, A. (2009). 1230 Acantilados con vegetación de las costas atlánticas y bálticas. En VV.AA., Bases ecológicas preliminares para la conservación de los tipos de hábitat de interés comunitario en España. Madrid: Ministerio de Medio Ambiente, y Medio Rural y Marino. 144 p.www. mapama.gob.es/es/biodiversidad/

Macías, F. (Ed.), (2014). Complejo básico-ultrabásico de Capelada-cabo Ortegal ¿Un nuevo geoparque para el desarrollo geoturístico y cultural de Galicia? IV Congreso ibérico de la ciencia del suelo. Andavira Editora. Santiago de Compostela. 98 pp.

Moreno, J.C. (Ed.), (2008). Lista Roja 2008 de la flora vascular española. Dirección General de Medio Natural y Política Forestal (Ministerio de Medio Ambiente, y Medio Rural y Marino, y Sociedad Española de Biología de la Conservación de Plantas), Madrid, $86 \mathrm{pp}$.

Pérez-Alberti, A. \& López-Bedoya, J. (2009). 8220 Laderas y salientes rocosos silíceos con vegetación casmofítica. En VV.AA., Bases ecológicas preliminares para la conservación de los tipos de hábitat de interés comunitario en España. Madrid: Ministerio de Medio Ambiente, y Medio Rural y Marino. 30 p. www. mapama.gob.es/es/biodiversidad/

Pinto da Silva, A.R. (1965). Os habitats serpentinicos e o seu racional aproveitamento agrario. En Consejo superior de investigaciones científicas (España). En A.
Pinto da Silva, (Ed.). Aportación de las Investigaciones ecológicas y agrícolas en la lucha del mundo contra el hambre: texto de las comunicaciones y discusiones. Madrid. 1- 40.

Pinto da Silva, A. R. (1970). A flora e a vegetação das áreas ultrabásicas do nordeste transmontano. Agron. Lusit., 30, 175-364.

Rivas-Martínez, S. (2004). Clasificación bioclimática de la Tierra. Versión 27-08-2004. http://www. globalbioclimatics.org/book/bioc/global_ bioclimatics_2.htm. Consultado 7 de noviembre, 2017.

Rivas-Martínez, S (2007). Mapa de series, geoseries y geopermaseries de vegetación de España (Memoria del mapa de vegetación potencial de España). Parte I. Itinera Geobot., 17, 5-435.

Rivas-Martínez S., Penas, A., Díaz, T.E., Río, S. del, Cantó, P., Hereros, L. \& Costa, J.C. (2014 a). Biogeography of Spain and Portugal. Preliminary typological synopsis. Internat. J. Geobot. Research. 4, 1-64.

Rivas-Martínez S., Izco, J., Díaz, T.E., Penas, A., Costa, J.C., Amigo, J., Herrero, L., Giménez de Azcárate, J. \& Río, S. del (2014b). The Galician-Portuguese Biogeographic Sector. An initial advance. Internat. J. Geobot. Research., 4, 65-81.

Rodríguez-Oubiña, S. \& Ortiz, S. (1991). Los pastizales pioneros vivaces de los suelos serpentínicos del NO ibérico. Lazaroa, 12, 333-344. 